\title{
Thermal disintegration of carbon fullerenes
}

\author{
B. L. Zhang, C. Z. Wang, C. T. Chan, and K. M. Ho \\ Ames Laboratory and Department of Physics and Astronomy, Iowa State University, Ames, Iowa 50011
}

(Received 1 March 1993)

\begin{abstract}
The process of thermal disintegration of carbon fullerenes is simulated by molecular dynamics using a tight-binding-force model. The most commonly observed fragments after the disintegration of carbon fullerenes are dimers, rings, and multiple rings. The fragmentation temperatures are obtained for fullerenes ranging from $\mathrm{C}_{20}$ to $\mathrm{C}_{90}$. The fragmentation temperature increases almost linearly with the cluster size for small cages $(n \leq 58)$, but becomes almost constant for larger fullerenes $(n=60$ and $n \geq 70)$. The effects of electronic entropy on the fragmentation temperature of fullerene are also discussed.
\end{abstract}

\section{INTRODUCTION}

$\mathrm{C}_{60}$ and the fullerene family exhibit various fascinating properties which have sparked enormous research interests from both theorists and experimentalists. ${ }^{1}$ In this paper, we present a systematic study of the stability of the carbon fullerenes against thermal disintegration. This is a first step towards establishing a "phase diagram" for the fullerenes as a function of cluster size and temperature. Since the fullerenes are produced in the high temperature processes, such as arc burning or laser vaporization of graphite, knowledge of the thermal stability of carbon cages is useful for controlling the synthesis of fullerenes. On the other hand, fullerenes possess a cagelike structure which spreads all the atoms over a closed surface. It is a system with both three-dimensional and two-dimensional characters. Therefore, understanding the process of the thermal disintegration of this unique system is also of interest from a basic scientific point of view. In our present simulations, we are studying systems at fairly high temperatures. To include the effects of electronic states at finite temperature, we used the Fermi-Dirac (FD) distribution to describe the occupation of the electronic states in the energy and force calculations in our tight-binding molecular-dynamics (TBMD) simulations. Our procedure follows closely those used in recent first-principles molecular-dynamics (FPMD) studies of metallic systems. ${ }^{2-4}$ It has been observed that the inclusion of electronic temperature effects not only avoids the instability caused by the change of occupancies of states near the Fermi level in the metallic systems, but also includes the electronic entropy in the calculation in a convenient manner. In this paper, we will report on the effect of considering the FD distribution on the specific physical property of thermal disintegration of fullerenes.

\section{METHOD}

Molecular-dynamics simulation is performed with a recently developed tight-binding model $^{5}$ for carbon systems. The total potential energy of the system is ex- pressed as

$$
E\left(\left\{\mathbf{r}_{i}\right\}\right)=\sum_{n}^{\text {occupied }}\left\langle\psi_{n}\left|H_{\mathrm{TB}}\left(\left\{\mathbf{r}_{i}\right\}\right)\right| \psi_{n}\right\rangle+E_{\mathrm{rep}}\left(\left\{\mathbf{r}_{i}\right\}\right) .
$$

The first term in (1) is the electronic energy calculated by the parametrized TB Hamiltonian $H_{\mathrm{TB}}\left(\left\{\mathbf{r}_{i}\right\}\right)$, and the second term is a short-ranged repulsive energy. All the parameters and functions were fitted to the results of first-principles local-density-functional calculations for the electronic band structures and binding energies of graphite, diamond, and the linear chain as a function of the carbon-carbon bond lengths. This tight-binding model has been quite successful in the study of structural and dynamical properties of carbon fullerenes, including the predictions of ground-state isomers of large fullerenes ${ }^{6}$ and vibrational properties of $\mathrm{C}_{60} \cdot{ }^{7}$ The fully relaxed $\mathrm{C}_{60}$ molecule obtained by the present scheme has icosahedral symmetry and bond lengths of $1.40 \AA$ and $1.46 \AA$, respectively, for the double and single bonds. These values agree very well with experimental results of $1.40 \AA$ and $1.45 \AA .^{8}$ Our tight-binding calculation yields a heat of formation of $0.40 \mathrm{eV}$ per atom relative to graphite and highest-occupied-molecular-orbital--lowestunoccupied-molecular-orbital (HOMO-LUMO) energy separation of $1.61 \mathrm{eV}$. These results also agree quite well with first-principles density-functional calculation results of $0.39 \mathrm{eV}$ per atom and $1.71 \mathrm{eV}$, respectively. ${ }^{9}$

Constant-temperature molecular-dynamics simulation is performed using simple velocity scaling for temperature control. The starting configurations are the groundstate fullerene structures obtained from our previous work. ${ }^{10,11}$ We study only the even-numbered clusters since odd-numbered clusters are observed to be much less abundant in the experimental mass spectra. Starting with the cluster in the ground-state fullerene structure, we gradually heat up the system at a rate of $500 \mathrm{~K}$ per 2000 steps $\left(0.7 \times 10^{-15}\right.$ sec. per step). When the system is close to the fragmentation point, we reduced the heating rate to $100 \mathrm{~K}$ per 2000 steps in order to have a more accurate estimate of the fragmentation temperature. The 


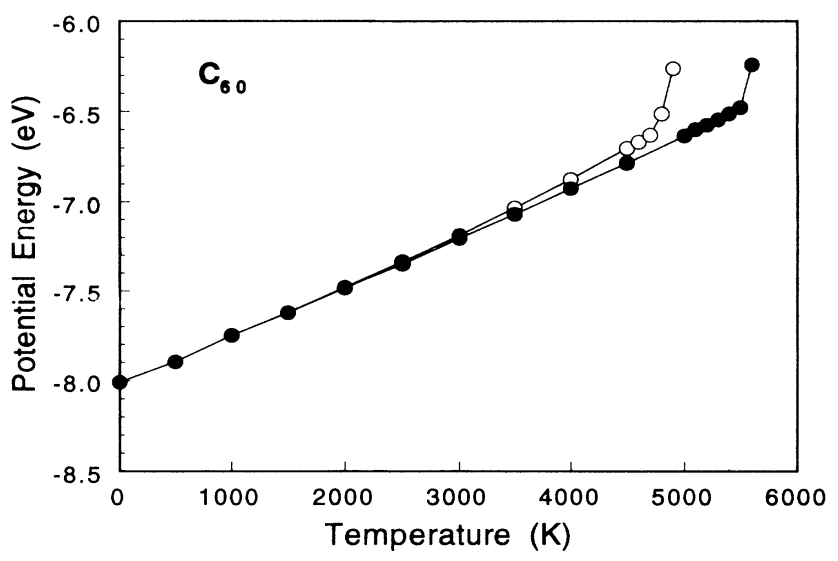

FIG. 1. The potential energy $\left(\mathrm{eV} /\right.$ atom) of $\mathrm{C}_{60}$ is plotted as a function of temperature, where the solid dots are the results without FD distribution and the open dots are the results with FD distribution.

potential energy of the system is monitored as a function of temperature, the results of a typical run are shown in Fig. 1. During the heating up process, there is a sudden jump in the potential energy at a certain temperature. By monitoring a graphic display of the atomic motions during the simulation, we verified that this corresponds to the onset of bond-breaking in the fullerene cage structure. This temperature is then recorded as the fragmentation temperature of the carbon fullerene. For $\mathrm{C}_{60}$, we also repeated the simulation using stochastic temperature control and obtained almost the same fragmentation temperature (within $100 \mathrm{~K}$ ) as the simple velocity scaling method.

In our previous TBMD simulations, ${ }^{6,10,11}$ the electrons always occupy the lowest energy states. In other words, the electron temperature $T_{\mathrm{el}}$ is set to zero. This treatment is acceptable when we are searching for the groundstate geometries when the temperature of the system is finally cooled down to zero. For carbon clusters with relatively large HOMO-LUMO gaps we expect this to be an accurate description at low temperatures. But in the present study, the clusters are warmed up to several thousand degrees. In the high temperature regime, the FD distribution is important because of the higher electronic temperature and narrower HOMO-LUMO gap caused by thermal vibrations. For $\mathrm{C}_{60}$, the HOMO-LUMO gap drops from $1.6 \mathrm{eV}$ at $T=0$ to $0.2 \mathrm{eV}$ at the fragmentation temperature which is about $5000 \mathrm{~K}\left(k_{B} T \sim 0.43 \mathrm{eV}\right)$. With the Fermi-Dirac distribution, the band structure energy and the Hellmann-Feynman force are obtained as follows:

$$
\begin{aligned}
E_{\mathrm{TB}} & =2 \sum_{i} \varepsilon_{i} f_{i}, \\
F_{\alpha}= & -2 \sum_{i}\left\langle\psi_{i}\left|\nabla_{\alpha} H_{\mathrm{TB}}\right| \psi_{i}\right\rangle f_{i} \\
& -2 \sum_{i} \varepsilon_{i} \frac{\partial f_{i}}{\partial\left(\varepsilon_{i}-\mu\right)} \nabla_{\alpha}\left(\varepsilon_{i}-\mu\right),
\end{aligned}
$$

where

$$
f_{i}=\frac{1}{e^{\left(\varepsilon_{i}-\mu\right) / k_{B} T_{\mathrm{el}}}+1}
$$

$\mu$ is the chemical potential and it is adjusted every time step to guarantee the conservation of the total number of electrons:

$$
2 \sum_{i} f_{i}=N_{\mathrm{el}}
$$

It was pointed out by Pederson and Jackson ${ }^{3}$ that it is very difficult to calculate the second term in Eq. (3) in the FPMD. However, Wentzcovitch, Martins, and Allen ${ }^{4}$ introduced the Mermin free energy: ${ }^{12}$

$$
\begin{aligned}
& \Omega=E_{\text {total }}+K_{I}-T_{\mathrm{el}} S \\
& S=-2 k_{B} \sum_{i}\left[f_{i} \ln f_{i}+\left(1-f_{i}\right) \ln \left(1-f_{i}\right)\right]
\end{aligned}
$$

and showed numerically that the FPMD simulation conserves the free energy $\Omega$ if one drops the second term in Eq. (3). It can be shown analytically that only the first term in (3) is required if the Hellmann-Feynman forces are calculated using the electronic free energy instead of electronic energy $E_{\mathrm{TB}}$. The second term in Eq. (3) is canceled by the derivative of the electronic entropy:

$$
\frac{\partial\left(T_{\mathrm{el}} S\right)}{\partial \alpha}=T_{\mathrm{el}} \sum_{i} \frac{\partial S}{\partial f_{i}} \frac{\partial f_{i}}{\partial\left(\varepsilon_{i}-\mu\right)} \nabla_{\alpha}\left(\varepsilon_{i}-\mu\right) .
$$

Equation (8) can be rewritten as:

$$
\frac{\partial\left(T_{\mathrm{el}} S\right)}{\partial \alpha}=-2 k_{B} T_{\mathrm{el}} \sum_{i} \frac{\partial\left[f_{i} \ln f_{i}+\left(1-f_{i}\right) \ln \left(1-f_{i}\right)\right]}{\partial f_{i}} \frac{\partial f_{i}}{\partial\left(\varepsilon_{i}-\mu\right)} \nabla_{\alpha}\left(\varepsilon_{i}-\mu\right)
$$

and after some simple algebra, Eq. (8) becomes

$$
\frac{\partial\left(T_{\mathrm{el}} S\right)}{\partial \alpha}=2 \sum_{i} \varepsilon_{i} \frac{\partial f_{i}}{\partial\left(\varepsilon_{i}-\mu\right)} \nabla_{\alpha}\left(\varepsilon_{i}-\mu\right)
$$

Here, we also used the conservation of the total number of electrons:

$$
\sum_{i} \frac{\partial f_{i}}{\partial\left(\varepsilon_{i}-\mu\right)} \nabla_{\alpha}\left(\varepsilon_{i}-\mu\right)=\frac{\partial}{\partial \alpha} \sum_{i} f_{i}=\frac{\partial}{\partial \alpha} N_{\mathrm{el}}=0
$$

Thus, the second term in Eq. (3) is canceled by the derivative of electronic entropy (8) and the first term is $-\nabla_{\alpha}\left(E_{\mathrm{TB}}-T_{\mathrm{el}} S\right)$. 
In our simulations, the electronic temperature is set to be equal to the desired ionic temperature.

\section{RESULTS AND DISCUSSIONS}

The fragmentation temperatures, with and without electronic temperature effects, are shown in Fig. 2 as a function of cluster size ranging from $\mathrm{C}_{20}$ to $\mathrm{C}_{90}$. The heat of formation of these fullerenes relative to graphite is plotted in Fig. 3 for the purpose of comparison. As a general trend, we find that the fragmentation temperature increase almost linearly with the cluster size in the range $\mathrm{C}_{20}-\mathrm{C}_{58}$. This trend is quite different from that of the formation energy (see Fig. 3) in which the slope decreases rapidly as the cage size increases. For clusters with more than 60 atoms, the fragmentation temperature is almost a constant, although their bonding energy keeps on increasing. We think that, while both the heat of formation and the fragmentation temperature measure the stability of the fullerene structure, the different behavior in the trends for these two properties can be understood if we take the fragmentation temperature to be a measure of the weakest bond in the cage, while the heat of formation is a measure of the average bonding in the cluster. Choosing $\mathrm{C}_{84}$ as an example, even though it has a larger heat of formation per atom than the $\mathbf{C}_{60}$ molecule, it has certain bonds weaker than the others due to the low-symmetry $\left(\mathrm{D}_{2}\right)$ structure. These weak bonds are not stronger than that of $\mathrm{C}_{60}$ and that is why the fragmentation temperatures of $\mathrm{C}_{84}$ and $\mathrm{C}_{60}$ are almost the same. Finally, it is worth noting that a sharp kink is found at $\mathrm{C}_{60}$ in both energy and fragmentation temperature plots, indicating its enhanced stability relative to its neighbors.

The criterion we used to determine the fragmentation point in this study may have overestimated the fragmentation temperature due to the rather short simulation times practicable in TBMD simulations. From our simulation, the time for redistributing the added energy among the different degrees of freedom is estimated to be about 5000 to 6000 steps. Our heating rate of

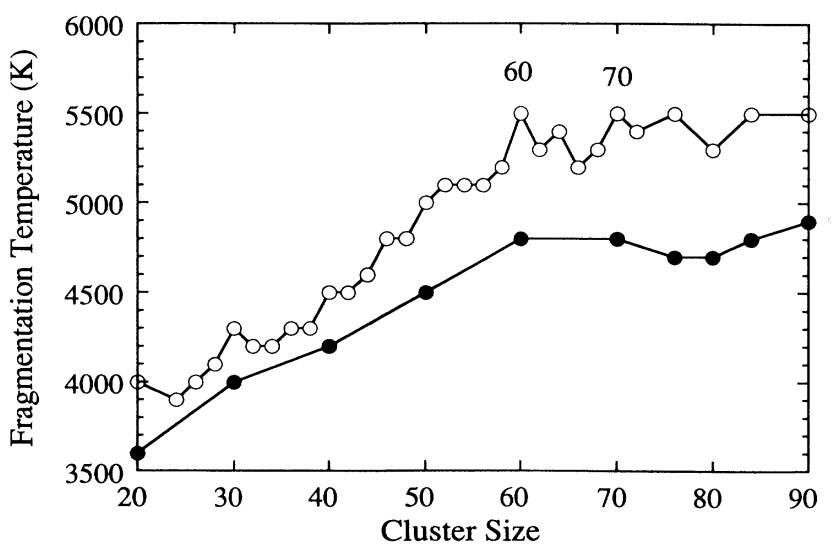

FIG. 2. The fragmentation temperature of fullerene is plotted as a function of cluster size, where the solid dots are the results with FD distribution and the open dots are the results without FD distribution.

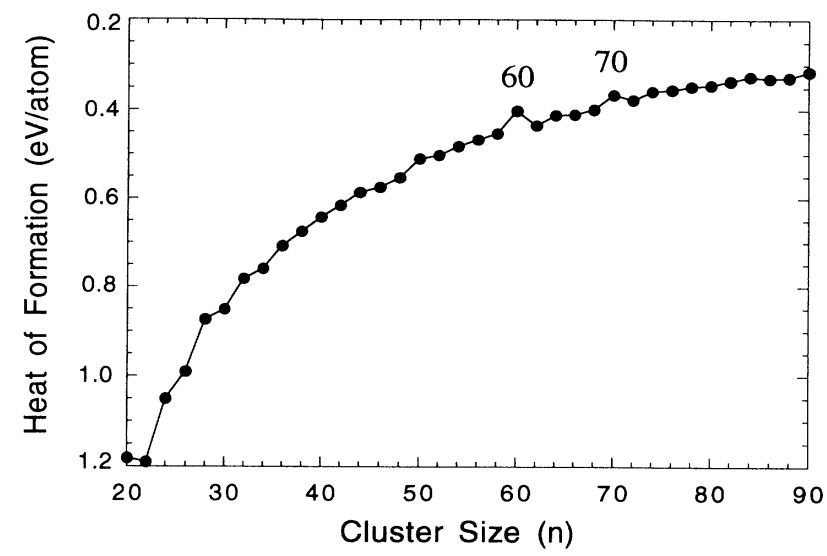

FIG. 3. The heat of formation ( $\mathrm{eV} /$ atom) of fullerene is plotted as a function of cluster size. (Note: the heat of formation is relative to bulk graphite.)

$100 \mathrm{~K}$ per 2000 steps near the disintegration region may therefore overestimate the disintegration temperature by about $200 \mathrm{~K}$. (The faster heating rate of $500 \mathrm{~K} / 2000$ steps far below the critical temperature will not have any significant effects on the disintegration temperature as long as the system is sufficiently thermalized at temperatures just below the disintegration temperature.) We have also performed longer time simulations for the thermal disintegration process of $\mathrm{C}_{60}$. We found that if we perform the simulation at $200 \mathrm{~K}$ lower than the disintegration temperature determined from the short simulation time (i.e., 2000 steps or $1.4 \mathrm{ps}$ ), the fullerene will break after 14000 steps (9.8 ps) of simulation. However, if we lower the temperature of the system by another $100 \mathrm{~K}$, the $\mathrm{C}_{60}$ molecule is found to be stable up to $50 \mathrm{ps}$ of simulation time. This result is consistent with the above analysis based on the energy redistribution process.

It should also be kept in mind that the present tightbinding model is fitted to reproduce the binding energy of first-principles density-functional calculation results, which overestimate the binding energy of graphite by about $10 \%$ in comparison with the experimental value. Thus there exists a corresponding systematic error in the fragmentation temperature if one wants to make a direct comparison with experimental results. Also, in the real experimental condition, the disintegration process can be influenced by collisions with other objects, such as atoms, dimers, and cluster fragments.

A schematic illustration of the fullerene fragmentation process is plotted in Fig. 4. In a typical disintegration process, after the onset of bond breaking, the atoms with dangling bonds stick out from the cage surface. These off-surface atoms vibrate much more violently than the rest of the network and the whole cage structure is finally unravelled into a ring or multiple-ring structure with the breaking off of fragments like dimers.

There is no noticeable difference in the disintegration process for simulations with and without including the FD distribution for the occupancy of electronic states except for a lowering of the fragmentation temperatures. As the electrons partially occupy the states with antibonding 


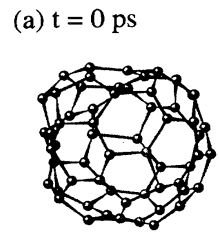

(b) $\mathrm{t}=0.07 \mathrm{ps}$
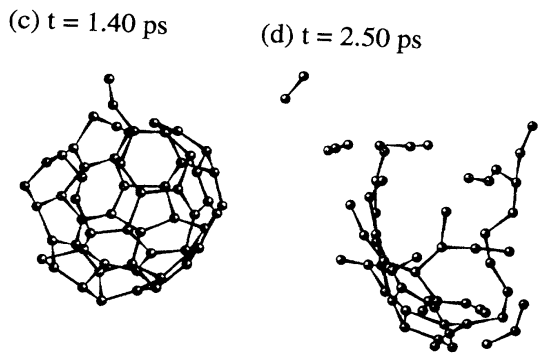

FIG. 4. Snapshots of the $\mathrm{C}_{60}$ fragmentation process at $T=5500 \mathrm{~K}$.

character above the Fermi level, the bonds between carbon atoms become weaker and the clusters break apart more easily. For $\mathrm{C}_{60}$, the HOMO-LUMO gap at $T=0 \mathrm{~K}$ is $1.6 \mathrm{eV}$, corresponding to $18500 \mathrm{~K}$, thus the occupancy above the Fermi level is negligible. But as the temperature increases, the structural distortions due to thermal vibrations cause the HOMO-LUMO gap to decrease rather quickly. The HOMO-LUMO gaps plotted in Fig. 5 are obtained from an average of 2000 time steps from the constant-temperature simulations. We can see that the HOMO-LUMO gap drops to around $0.25 \mathrm{eV}$ near the fragmentation temperature $5000 \mathrm{~K}$. In Fig. 1, we show the potential energy of $\mathrm{C}_{60}$ as a function of temperature for the simulations with and without Fermi-Dirac distribution. Since $\mathrm{C}_{60}$ has a large HOMO-LUMO gap, these two curves match exactly for low temperatures. But after the temperature exceeds $2500 \mathrm{~K}$, there is a significant difference between the two curves. The curve with zero electronic temperature is almost linear, indicating nearly harmonic thermal vibrations, while the curve with nonzero electronic temperature increases faster. Thus temperature effects for the electronic system enhance the anharmonic effects at high temperatures and this may also affect other dynamical properties, such as shifts and broadening of the vibration frequencies of the molecule. In the present study, the effect on fragmentation tem-

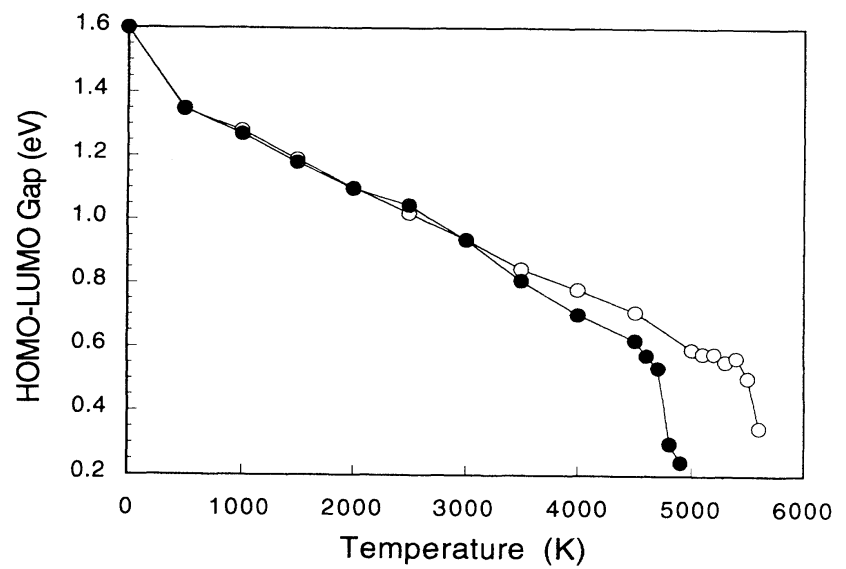

FIG. 5. The HOMO-LUMO gap of $\mathrm{C}_{60}$ is plotted as a function of temperature where the solid dots are the results with FD distribution and the open dots are the results without FD distribution.

peratures is less than $10 \%$ because of the relatively large HOMO-LUMO gaps in most of the carbon clusters.

\section{CONCLUSIONS}

In summary, we performed tight-binding moleculardynamics simulations of the thermal disintegration of fullerene cages. We found that the fragmentation temperature is sensitive to the cluster size for small clusters but almost constant for larger clusters. With the implementation of the Fermi-Dirac distribution for the occupation of the electronic states, the fragmentation temperatures of carbon fullerenes are reduced by about $10 \%$ due to the occupation of antibonding states at $T_{\text {el }} \neq 0$. From this study, we found that Fermi-Dirac distribution of electronic states also enhances the anharmonicity of the carbon clusters and this might be important for studying the dynamics of systems that have smaller or no band gaps at high temperatures.

\section{ACKNOWLEDGMENTS}

Ames Laboratory is operated for the U.S. Department of Energy by Iowa State University under Contract No. W-7405-ENG-82. This work was supported by the Director of Energy Research, Office of Basic Energy Sciences, including a grant of computer time at NERSC at Livermore.
${ }^{1}$ R. E. Smalley, Acc. Chem. Res. 78, 98 (1992), and references therein.

${ }^{2}$ G. Fernando, G.-X. Qian, M. Weinert, and J. D. Davenport, Phys. Rev. B 40, 7985 (1989); G.-X. Qian, M. Weinert, G. Fernando, and J. D. Davenport, Phys. Rev. Lett. 64, 1146 (1990).

${ }^{3}$ M. Pederson and K. Jackson, Phys. Rev. B 43, 7312 (1991).

${ }^{4}$ R. M. Wentzcovitch, J. L. Martin, and P. B. Allen, Phys. Rev. B 45, 11372 (1992).

${ }^{5}$ C. H. Xu, C. Z. Wang, C. T. Chan, and K. M. Ho, J. Phys. Condens. Matter 4, 6047 (1992).

${ }^{6}$ B. L. Zhang, C. Z. Wang, and K. M. Ho, Chem. Phys. Lett.
193, 225 (1992); X. Q. Wang, C. Z. Wang, B. L. Zhang, and K. M. Ho, Phys. Rev. Lett. 67, 67 (1992).

${ }^{7}$ C. Z. Wang, C. H. Xu, C. T. Chan, and K. M. Ho, J. Phys. Chem. 96, 7603 (1992).

${ }^{8}$ C. S. Yannoni, P. P. Bernier, D. S. Bethune, G. Meijer, and J. R. Salem, J. Am. Chem. Soc. 113, 3190 (1991).

${ }^{9}$ J. L. Martins (private communication).

${ }^{10}$ B. L. Zhang, C. Z. Wang, K. M. Ho, C. H. Xu, and C. T. Chan, J. Chem. Phys. 97, 5007 (1992).

${ }^{11}$ B. L. Zhang, C. Z. Wang, K. M. Ho, C. H. Xu, and C. T. Chan, J. Chem. Phys. 98, 3095 (1993).

${ }^{12}$ N. D. Mermin, Phys. Rev. 137, A1441 (1965). 